\title{
Investigation of orientation effects in films of nitric acid trihydrate
}

\author{
Belén Maté, ${ }^{* a}$ Ismael K. Ortega ${ }^{a b}$ Miguel A. Moreno, ${ }^{a}$ Rafael Escribano ${ }^{a}$ \\ and Víctor J. Herrero* ${ }^{* a}$
}

\author{
${ }^{a}$ Instituto de Estructura de la Materia (CSIC), Serrano 123, Madrid 28006, Spain. \\ E-mail:bmate@iem.cfmac.csic.es; vherrero@iem.cfmac.csic.es \\ ${ }^{b}$ Departamento de Quimica Física y Analitica, Universidad de Jaén, Paraje Las Lagunillas, \\ Jaén 23071, Spain
}

Received 19th February 2004, Accepted 6th May 2004

First published as an Advance Article on the web 10th June 2004

\begin{abstract}
An investigation of orientation effects in films of nitric acid trihydrate (NAT) is presented, based on a systematic study of reflection-absorption infrared spectra of samples of varying thickness. The samples are prepared by vapor deposition on an Al substrate at $90 \mathrm{~K}$ and annealing at $175 \mathrm{~K}$ to produce crystalline $\alpha$-NAT films. Spectra were recorded at a grazing angle of $75^{\circ}$, with polarized radiation. The observed spectra are compared with predictions of a classical Fresnel model, in order to test the available optical indices of NAT, which are of great importance for the accurate interpretation of data from remote sensing measurements. Whereas a similar procedure yielded satisfactory results for water ice, it is found that the agreement is not acceptable for NAT crystals. An explanation is suggested in terms of anisotropic or birefringent properties of the crystals, or as due to a preferential alignment of the films. The infrared activity of a peak at $\approx 1140 \mathrm{~cm}^{-1}$, which had previously been associated to changes in the crystal structure upon heating, is here explained in terms of a rearrangement of the polycrystals in the sample.
\end{abstract}

\section{Introduction}

The assessment of the role of nitric acid trihydrate, NAT, in the physical chemistry of polar stratospheric clouds (PSCs) has stimulated many works dedicated to the investigation of its structure and properties over the last decade (see refs. 1-5 and references therein).

Infrared spectroscopy has been one of the main techniques used for the structural characterization and for thermodynamic and kinetic studies of phase transitions and chemical reactions involving NAT. Transmission IR spectra attributed to crystalline NAT were published by different groups at the beginning of the nineties. ${ }^{6-10}$ Empirical assignments of the IR spectra, based on the available spectroscopic information on the individual molecules or ions present in the NAT structure $^{11,12}$ were also proposed in several works. ${ }^{7,8,13,14}$ The initial controversy about the actual degree of hydration of the samples attributed to $\mathrm{NAT}^{7,8}$ was settled by Koehler et al. ${ }^{10}$ who performed simultaneously IR spectroscopy and thermal programmed desorption measurements with mass spectrometric detection of the products, and provided a link between the stoichiometry of the deposited samples and the corresponding IR spectra. In addition, these authors noted that the IR spectrum of NAT changed upon heating the sample above $\approx$ $185 \mathrm{~K}$ and attributed it to a different phase of NAT. They termed the lower temperature phase $\alpha$-NAT and the higher temperature phase $\beta$-NAT.

Empirical optical constants for the solid nitric acid hydrates were derived by Toon et al. ${ }^{15}$ over the $4000-500 \mathrm{~cm}^{-1}$ range from transmission spectra of films of different thickness deposited on a transparent substrate. These optical constants were used for the simulation of IR spectra measured on PSCs over Antarctica under conditions excluding the presence of water ice. ${ }^{16,17}$ The simulations did not allow the identification of crystalline NAT. ${ }^{18}$ Later field measurements performed on particles of PSCs above the ice frost point and based on sophisticated mass spectrometric techniques led to $3: 1$ water to nitric acid molecular ratios, consistent with the expected presence of solid NAT. ${ }^{2}$

Several groups carried out IR extinction measurements on laboratory generated NAT aerosols. ${ }^{19-22}$ These experiments reproduced more closely the actual stratospheric conditions and provided an unsupported solid sample, whose formation is not influenced by the presence of a substrate. Richwine et al. ${ }^{21}$ determined the IR complex refractive indices of crystalline $\alpha$-NAT between 4000 and $700 \mathrm{~cm}^{-1}$ from such aerosol spectra using a Mie scattering model. The optical indices thus obtained did not differ much from those based on thin layer measurements, ${ }^{15}$ although some discrepancies were found, especially in the wavenumber region below $\approx 1500 \mathrm{~cm}^{-1}$.

Tisdale t $_{\text {al }}{ }^{23}$ recorded transmission IR spectra of thin films of $\alpha$-NAT deposited as amorphous solids and annealed at different temperatures between 162 and $180 \mathrm{~K}$. Appreciable differences were found between the spectra corresponding to samples crystallized below or above $175 \mathrm{~K}$. The two sets of optical constants commented on in the previous paragraphs ${ }^{15,21}$ were used for the simulations of the data, and it was found that the spectra of thin layers crystallized at $162 \mathrm{~K}$ were better reproduced with the optical indices obtained for aerosols at $175 \mathrm{~K}^{21}$ than with those derived from thin films at $181 \mathrm{~K}^{15}$ The authors concluded that the conditions of formation and in particular the temperature, could be more relevant than the type of sample (thin film or aerosol) in determining the spectrum of NAT. As possible explanations for the observed differences it was suggested that the lower temperature samples might not contain enough energy for the crystal to form a perfect defect-free lattice. A second alternative also suggested by these authors is that the presumably birefringent NAT crystal, ${ }^{24}$ when formed on a substrate, would tend to align preferentially along a given optical axis, the alignment being more complete for higher annealing temperatures.

The availability of reliable optical indices with a well-established range of validity is of great importance for the interpretation of field data from atmospheric observations (see 
ref. 25 and references therein), and has been one of the reasons that have prompted the present investigation, in view of the apparently unsolved queries mentioned above.

The technique of reflection-absorption infrared spectroscopy (RAIRS) is especially well suited to the investigation of the possible orienting effects of a substrate on the spectral features of a deposited sample, since one can take advantage of the dependence of the spectra on the reflection-absorption geometry, on the polarization of the incident light, and on the thickness of the deposited layer. ${ }^{26-28}$ From a comparison of transmission and RAIR spectra, Sodeau and coworkers ${ }^{14}$ concluded that very thin $(\approx 100 \mathrm{~nm})$ films of NAT crystals formed on a gold foil tended to be oriented with the $C_{3}$ symmetry axis of the nitrate and hydronium ions perpendicular to the substrate surface. In a recent work ${ }^{29}$ we have reported a detailed RAIRS study of vapor deposited water-ice layers with thickness ranging from $100 \mathrm{~nm}$ to more than $5 \mu \mathrm{m}$, using both polarized and non-polarized incident IR radiation. Except for some sharp peaks in the spectra recorded with polarized light and mostly associated with interferences, the whole set of spectra could be well reproduced with a simple model based on Fresnel equations ${ }^{27}$ and with empirical optical indices from the literature. These results and the good agreement previously obtained by Mitlin and Leung ${ }^{30}$ on similar experiments, strengthened the confidence in the validity of the model for the simulation of RAIR spectra even of relatively thick films.

RAIR spectra of crystalline NAT have been recorded by various groups as a part of their research, ${ }^{3,13,14,31-36}$ but as far as we know no systematic experimental investigation of the reflection-absorption spectroscopy of this substance has been performed to date. Motivated by the above mentioned question about optical indices, involving a possible anisotropy in the optical properties of NAT, and also possible orienting effects of the substrate on the deposited layers, we have undertaken the present investigation, in which we present polarized and non-polarized RAIR spectra of $\alpha$-NAT films with thickness between $\approx 370 \mathrm{~nm}$ and $2 \mu \mathrm{m}$. The experimental measurements are compared to Fresnel model simulations, and the observed spectral features are discussed in terms of the crystal structure and of the theoretical assignment of the crystal spectrum reported previously. ${ }^{37}$

\section{Experimental}

The experimental system used in the present work is described elsewhere ${ }^{29,38,39}$ and only the relevant details will be given here. The experiment was conducted in a UHV cylindrical chamber evacuated by a turbomolecular pump to a base pressure lower than $3 \times 10^{-8}$ mbar, provided with a liquid nitrogen Dewar in contact with the deposition substrate. After filling the Dewar the base pressure was in the $10^{-9}$ mbar range. The cold deposition surface was made of polished aluminum. The surface temperature could be regulated between 85 and $323 \mathrm{~K}$ with an accuracy of $1 \mathrm{~K}$.

In a previous work ${ }^{36}$ mixed vapors of water and nitric acid in equilibrium with the corresponding $\mathrm{HNO}_{3}$ solutions were introduced into the chamber through a regulation needle valve in order to prepare thin films of the various $\mathrm{HNO}_{3}$ hydrates. However this procedure led to instabilities in the vapor composition; therefore in the present experiments two independent inlets, for water and nitric acid, were used to introduce the vapors. In this way the relative concentrations of water and nitric acid in the chamber could be precisely controlled. To obtain a uniform deposition during the production of the films, the gas inlets were not directed to the substrate surface, but deflected to the wall so that the whole chamber was filled homogeneously with a small pressure of the vapors of interest. Changing the ratio $\mathrm{H}_{2} \mathrm{O}: \mathrm{HNO}_{3}$ in the vapor and/or changing the temperature of the aluminum surface, produced films with different stoichiometries. To obtain $\alpha$-NAT films the aluminum surface was maintained at $90 \mathrm{~K}$ and the vacuum chamber was backfilled with $2 \times 10^{-5}$ mbar of $\mathrm{H}_{2} \mathrm{O}$ and $1.2 \times 10^{-5}$ mbar of $\mathrm{HNO}_{3}$ (see below for calibration of the absolute pressures). Under these conditions the collision rate of water molecules with the deposition surface is three times larger than that of $\mathrm{HNO}_{3}$ molecules, and the sticking coefficient can be taken approximately as one for both substances. The deposition time ranged between 3 and $16 \mathrm{~min}$ and the thickness, $d$, of the growing films was monitored by measuring the interference fringes of a $\mathrm{He}-\mathrm{Ne}$ laser, as described in ref. 29. A refraction index of 1.43 was taken for the 3:1 water:nitric acid amorphous deposit. ${ }^{40}$ Typical growth rates of $\approx 3 \mathrm{~nm} \mathrm{~s}^{-1}$ were obtained. The samples were then annealed at $175 \mathrm{~K}$ and thus transformed to crystalline films of $\alpha$-NAT. Under the generation conditions described, the crystalline samples should be formed by polycrystals. RAIR spectra were recorded at regular intervals during the annealing. After $20 \mathrm{~min}$, when the spectral shapes had stabilized, the films were cooled again at $90 \mathrm{~K}$ before recording the spectra. In the course of this process some sample was desorbed. This desorption led also to the appearance of an interference pattern in the reflected $\mathrm{He}-\mathrm{Ne}$ laser signal which was used to evaluate the amount of lost sample and the approximate thickness of the crystalline films. Uncertainty in the $d$ values thus determined is estimated to be $\pm 15 \%$. Using the same procedure a spectrum of a thin film deposited at $150 \mathrm{~K}$ and then annealed at $180 \mathrm{~K}$ was also measured. The comparison will be discussed below.

The calibration of the $\mathrm{H}_{2} \mathrm{O}$ and $\mathrm{HNO}_{3}$ pressures was made indirectly by depositing films of pure water and pure $\mathrm{HNO}_{3}$ at $90 \mathrm{~K}$. The growth rates of these films were also monitored by recording the interference fringes of a $\mathrm{He}-\mathrm{Ne}$ laser. For the estimate of the film thickness, refractive indices of 1.27 and 1.49 were taken for water ${ }^{41}$ and nitric acid ${ }^{40}$ respectively. The growth rate as a function of the gas pressure in the deposition chamber, $P_{\mathrm{g}}$, can be estimated from gas kinetic theory by assuming a sticking coefficient of 1 (which is reasonable for $90 \mathrm{~K}$ ):

$$
\frac{\mathrm{d} x}{\mathrm{~d} t}=\frac{P_{g}}{\rho \sqrt{2 \pi m_{g} k T_{g}}}
$$

where $T_{\mathrm{g}}$ is the temperature of the gas of interest (water or $\mathrm{HNO}_{3}$ ) in the chamber, $\rho$ is the density of the solid at $90 \mathrm{~K}$ $\left(0.82 \mathrm{~g} \mathrm{~cm}^{-3}\right.$ for water ${ }^{41}$ and $1.85 \mathrm{~g} \mathrm{~cm}^{-3}$ for nitric acid $\left.{ }^{42}\right), m_{\mathrm{g}}$ is the mass of the gas phase molecules and $k$ the Boltzmann constant. Calibration of the corresponding regulating valve for the measurement of absolute pressures is achieved by equating the growth rate of eqn. (1) to the value obtained from the interference measurements. The estimated uncertainty in $P_{\mathrm{g}}$ is $\approx 20 \%$.

A FTIR spectrometer Brucker IFS66 was used to record the spectra. The IR radiation was focused on the sample with a $\mathrm{KBr}$ lens at an incidence angle of $75^{\circ}$. The reflected IR light was focused by a curved mirror onto a Mercury Cadmium Telluride (MCT) detector cooled with liquid nitrogen. A polarizer SPECAC KRS-5 placed before the focusing lens was used to select the incident radiation with an electric field vector perpendicular (s) or parallel (p) to the plane of incidence, in order to record the corresponding s- or p-polarized spectra. Each spectrum was obtained from the addition of two series of 512 scans, recorded at $8 \mathrm{~cm}^{-1}$ apodized resolution. In analogy with ref. 29 all the spectra are presented in absorbance units corresponding to base 10 logarithms.

A model based on Fresnel equations already described in previous papers ${ }^{27,29}$ was used to simulate the $\alpha$-NAT experimental RAIRS spectra. The two sets of empirical complex refractive indices of $\alpha$-NAT available in the literature, derived from transmission spectra of thin films deposited at $181 \mathrm{~K}^{15}$ and from extinction spectra of aerosols at $175 \mathrm{~K},{ }^{21}$ were used for the simulations. As mentioned in the introduction, both sets of indices present a good qualitative agreement, but 
significant differences appear in the frequency region below $\approx 1500 \mathrm{~cm}^{-1}$. The wavelength dependent optical indices for metallic $\mathrm{Al}$ were taken from the room temperature values of Palik. ${ }^{43}$ In every case, we have used in the simulations the $d$ value giving the best fit to the measured spectra. Simulations were also performed for the experimental conditions (metallic substrate, angle of incidence) of other groups who have reported RAIR spectra of $\alpha$-NAT. In some of these experiments gold was used as a substrate and the corresponding optical indices were also taken from ref. 43.

\section{Results}

Fig. 1 shows s-polarized (S), p-polarized (P) and non-polarized (T) spectra of crystalline $\alpha$-NAT films. Fig. 2 shows an enlargement of the spectral region below $2000 \mathrm{~cm}^{-1}$ for films of thickness lower than $1 \mu \mathrm{m}$. The corresponding Fresnel model simulations using the refractive indices of Toon et $a l .{ }^{15}$ or Richwine et al. ${ }^{21}$ are also presented in the figure for comparison. All the spectra considered show a broad absorption, partially resolved into two peaks, in the $3500-3000 \mathrm{~cm}^{-1}$ region, which corresponds mostly to the $\mathrm{O}-\mathrm{H}$ stretching vibrations of the water molecules. ${ }^{7,37}$ The lower wavenumber end of this region is usually assigned to water molecules with a stronger hydrogen bonding. In the s-polarized spectra of the 600 and $800 \mathrm{~nm}$ films a broad absorbance maximum is seen between 2000 and $3000 \mathrm{~cm}^{-1}$. This maximum, whose location shifts with layer thickness, is not due to

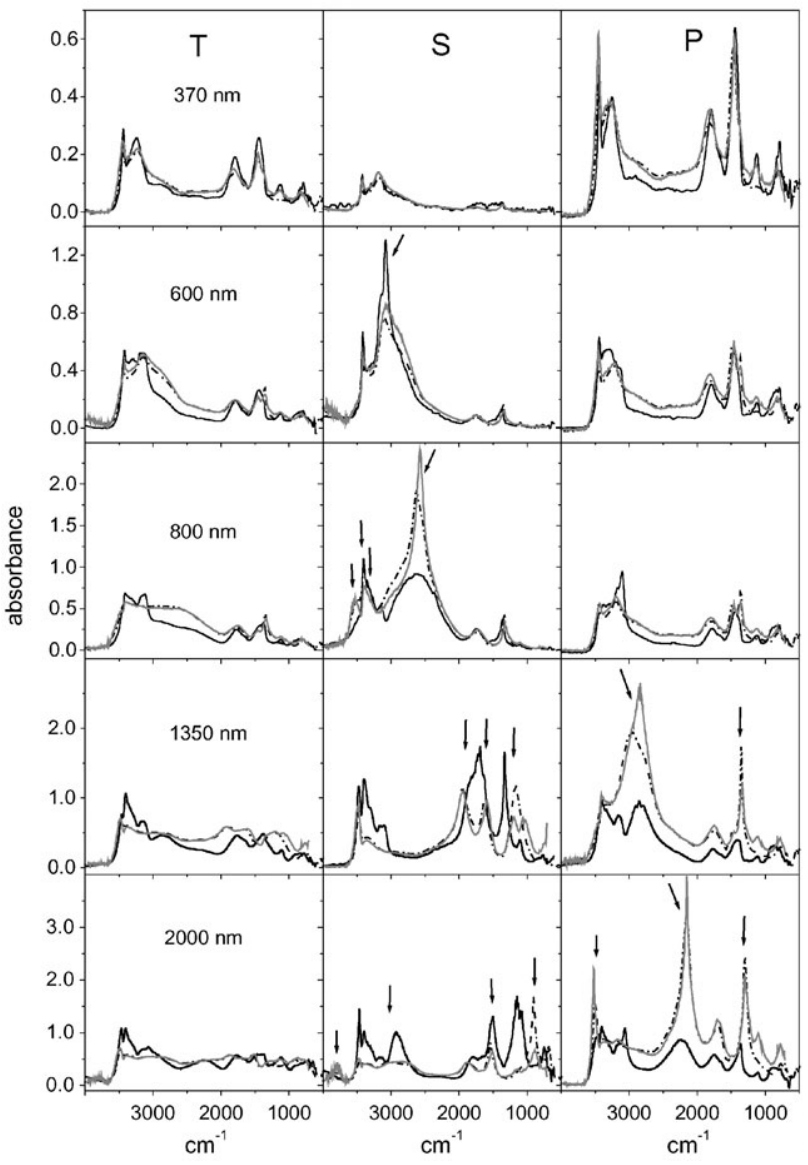

Fig. 1 Grazing angle $\left(\theta=75^{\circ}\right)$ RAIR spectra of NAT films with thickness values up to $2 \mu \mathrm{m}$ formed by deposition at $90 \mathrm{~K}$ and annealing at $175 \mathrm{~K}$. Solid trace: experimental measurements recorded with IR light non-polarized (T), and polarized parallel (S) or perpendicular $(\mathrm{P})$ to the plane of incidence. Dash-dot trace: simulations of the spectra with a Fresnel model (see text and refs. 27 and 29) and with the optical indices of Toon et al. ${ }^{15}$ Gray trace: simulations with the optical indices of Richwine et al. ${ }^{21}$ Arrows indicate the position of the IR interferences (see Table 1).

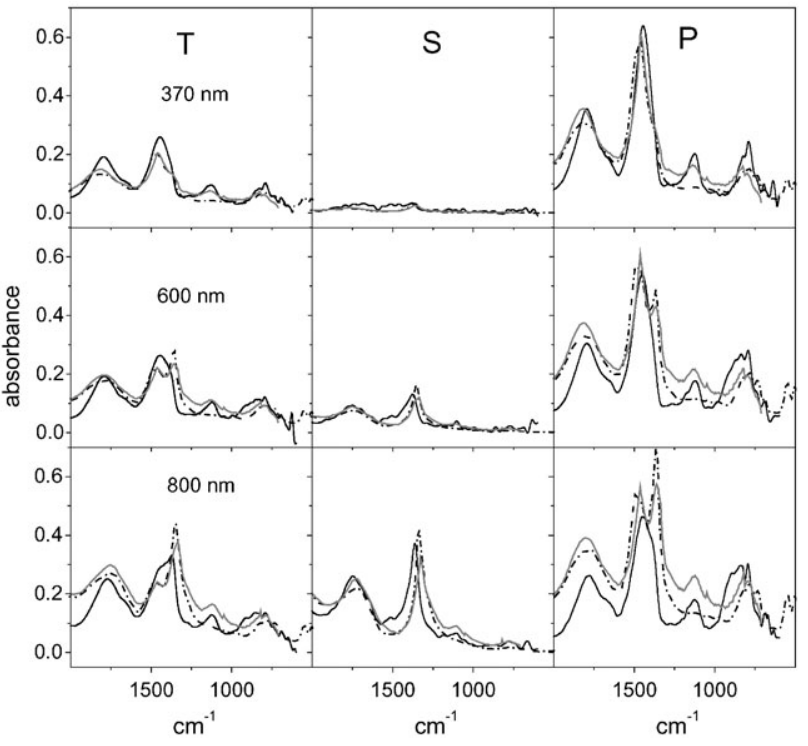

Fig. 2 Enlargement of the spectral region below $2000 \mathrm{~cm}^{-1}$ of Fig. 1, for films of thickness lower than $1 \mu \mathrm{m}$.

sample absorptions, but to destructive IR interferences that lead to a decrease in the radiation arriving to the detector and show up as bands in the absorption spectra. These interferences are marked with an arrow and will be commented on below. Another group of peaks corresponding essentially to vibrations of nitrate and hydronium ions ${ }^{7,37}$ appears at frequencies below $2000 \mathrm{~cm}^{-1}$.

Fig. 1 shows that the s-polarized spectrum of the thinnest film $(370 \mathrm{~nm})$ has a much lower overall intensity than the corresponding p-polarized spectrum, having just a very weak absorbance in the lower frequency region. This effect is typical of the grazing angle RAIRS technique used for the measurements. In the close vicinity of the substrate surface the p-polarization component of the incident radiation is enhanced upon reflection whereas the s-polarization component is cancelled out by the metal surface selection rule (MSSR). ${ }^{26,28}$ With increasing layer thickness, the effects of the metal surface turn out to be gradually smaller. The s-polarized spectrum becomes progressively stronger and the region below $2000 \mathrm{~cm}^{-1}$ is better resolved.

The Fresnel model simulations are in good agreement with the experimental s-polarized spectrum of the $370 \mathrm{~nm}$ film, whereas some discrepancies are appreciable for the p-polarized spectrum. The p-polarized calculated spectra with both sets of indices overestimate the absorbance in the $3000-2000 \mathrm{~cm}^{-1}$ range. More interestingly, while the location of all the peaks is well accounted for by the optical indices of Richwine et al., ${ }^{21}$ the simulation with the indices of Toon et al. ${ }^{15}$ fails to reproduce the peak at $\approx 1140 \mathrm{~cm}^{-1}$; this peak is missing in all the simulations performed with this set of indices. This fact had already been observed and discussed by Tisdale et al. ${ }^{23}$ The simulation of the spectrum recorded with non-polarized radiation leads to a reasonable agreement in the overall spectral shape, with some differences in the magnitude of the absorbance, which is underestimated at the peaks and slightly overestimated in the almost flat region between 2000 and $3000 \mathrm{~cm}^{-1}$. For films with $d<1 \mu \mathrm{m}$ (upper three rows in Fig. 1), the agreement between experiment and simulation deteriorates with growing thickness in the p-polarized and non-polarized spectra, with calculated absorbances tending to be larger than the observed ones over most of the spectral range. In the s-polarized spectra, the accordance is worst in the region of the strong interferences, which is not altogether surprising since the model calculations assume flat reflecting surfaces and the magnitude of the absorbance at the wavenumber of the interference maxima is very sensitive to 
the roughness of the real surfaces. For wavenumbers lower than $2000 \mathrm{~cm}^{-1}$, outside the region disturbed by interferences, the simulated spectra are in fairly good agreement with the observations.

In the spectra of the two thickest layers studied $(d>1 \mu \mathrm{m}$, two bottom rows in Fig. 1), the main absorptions described in the previous paragraph are still recognizable. However, for these films molecular absorptions are largely obscured by interference peaks over the whole spectral range. In our former study on thick films of water ice, ${ }^{29}$ the RAIR spectral shapes, including interference peaks, could be approximately reproduced by the Fresnel model even for layers having widths larger than $5 \mu \mathrm{m}$. In the present case with $\alpha$-NAT films with $d \leq 2 \mu \mathrm{m}$, the model cannot account satisfactorily for the observations with either of the two sets of refraction indices available. ${ }^{15,21}$ The location of the interference peaks depends on the layer thickness, on the angle of incidence and on the real part of the refractive index, and can be estimated approximately as indicated in refs. 29 and 44. Table 1 lists the wavenumber corresponding to maxima of IR destructive interferences relevant to our NAT films, calculated with the indices of Toon et al., ${ }^{15}$ which are also pointed out by arrows in Fig. 1. As seen in this figure, the intensity of the interferences in the p-polarized spectra, where they nearly coincide with molecular absorptions, is largely enhanced.

In a previous work, we reported the RAIR spectrum of an $\alpha$-NAT film measured in our laboratory with non-polarized radiation. ${ }^{36}$ The thickness of the film was not known with precision, but a rough estimate from exposure with pressure readings from an uncalibrated ionization gauge, led to values of the order of microns. A comparison of that spectrum with those of Fig. 1 indicates that the actual thickness of that film was smaller than $1 \mu \mathrm{m}$, and that the sample contained probably water in excess.

\section{Discussion}

\section{Orientation effects}

The imaginary part of the complex refractive indices available in the literature was derived from absorption spectra of either thin films ${ }^{15}$ at $181 \mathrm{~K}$ or small aerosols ${ }^{21}$ at $175 \mathrm{~K}$, and for both sets the real part was estimated indirectly via a KramersKronig transform using some modeling and scaling which imply isotropic media. The differences between the results of the two groups are not too large overall. The influence of the nature of the NAT sample on the derived indices, and, in particular the possibility that the deposition substrate might induce an orientation on the crystalline layers, are still subjects of debate. ${ }^{14,21,23,45}$ It is therefore possible that neither of the two sets of optical constants is precise enough for the

Table 1 Calculated wavenumber position of maxima of destructive IR interferences indicated by arrows in the $\alpha$-NAT spectra of Figs. 1 and $2^{a}$

\begin{tabular}{llllll}
\hline Pol-m & $d / \mu \mathrm{m}$ & calc. $/ \mathrm{cm}^{-1}$ & Pol-m & $d / \mu \mathrm{m}$ & calc. $/ \mathrm{cm}^{-1}$ \\
\hline S-0 & 0.6 & 3140 & S-0 & 2 & 905 \\
S-0 & 0.8 & 2630 & S-0 & 2 & 1525 \\
S-0 & 0.8 & 3220 & S-1 & 2 & 3140 \\
S-0 & 0.8 & 3410 & S-1 & 2 & 3800 \\
S-0 & 0.8 & 3600 & P-1 & 2 & 1360 \\
S-0 & 1.35 & 1180 & P-1 & 2 & 2200 \\
S-0 & 1.35 & 1610 & P-1 & 2 & 3495 \\
S-0 & 1.35 & 1880 & & & \\
P-1 & 1.35 & 3000 & & & \\
P-1 & 1.35 & 1350 & & &
\end{tabular}

${ }^{a}$ Pol-m: polarization of the IR radiation and interference order; $d$ : NAT film thickness; calc.: calculated wavenumber with the Fresnel model and optical indices of Toon et al. ${ }^{15}$ simulation of the RAIR spectra, which are sensitive to both components of the complex refactive index, ${ }^{26,27,29}$ especially if the NAT crystals exhibit a high degree of optical anisotropy, and if the crystalline sample is indeed oriented. According to the X-ray data, ${ }^{11,12} \beta$-NAT crystals have orthorhombic symmetry with unit-cell parameters $a=9.48 \AA, b=14.68 \AA$ and $c=3.43 \AA$. In a preliminary measurement carried out at $248 \mathrm{~K}$ on large $\beta$-NAT crystals obtained from a $3: 1 \mathrm{H}_{2} \mathrm{O}-\mathrm{HNO}_{3}$ solution, Kooshmanian et al. ${ }^{24}$ determined appreciably different values for the three principal (real) indices of refraction of $\beta$-NAT along the crystallographic axes $\left(n_{\mathrm{a}}\right.$ or $\mathrm{b}=1.38 \pm 0.01$, $\left.n_{\mathrm{b} \text { or a }}=1.59 \pm 0.01, n_{\mathrm{c}}=1.48 \pm 0.01\right)$, at the wavelength of a $\mathrm{He}-\mathrm{Ne}$ laser $(632.8 \mathrm{~nm})$. In a previous experiment, Middlebrook et al..$^{40}$ had measured an index of refraction for each of the two polymorphs ( $\alpha$ and $\beta$ ) of vapor deposited NAT. For the derivation of these data the authors used a $\mathrm{He}-\mathrm{Ne}$ laser at near normal incidence with respect to the NAT film and recorded the optical interferences as a function of film growth. The values thus obtained were $n=1.51 \pm 0.01$ and $n \geq 1.46$ for $\alpha$ - and $\beta$-NAT respectively. In that work, it was tacitly assumed that the polycrystals in the film were randomly oriented and that the refraction indices corresponded to a directional average; the likelihood of a preferential alignment of the crystallographic axes with respect to the deposition surface was not discussed. However, a comparison of the two experiments shows that the indices of the $\alpha$ - and $\beta$-NAT films are very close to the $n_{\mathrm{c}}$ value of Kooshmanian et al. ${ }^{24}$ and suggests that the experiment of Middlebrook et al. ${ }^{40}$ might have sampled predominantly the $c$ axis of the crystal, which would imply an approximate orientation of this axis perpendicular to the substrate.

A comparison between the s- and p-polarized RAIR spectra of Fig. 2 could provide in principle information about orientation in the crystalline layers. To avoid the distortion caused by IR interferences the comparison will be restricted to wavenumbers lower than $2000 \mathrm{~cm}^{-1}$. In this spectral range, the p-polarized spectra present four absorption peaks with maxima at about 1790, 1440, 1130 and $800 \mathrm{~cm}^{-1}$. The intensity of these peaks is similar for the three layers considered. In contrast, the s-polarized spectra show the emergence of two peaks with maxima at $\approx 1730$ and $1370 \mathrm{~cm}^{-1}$ that increase in intensity with growing film thickness, due to the gradual relaxation of the MSSR. The existence of absorption features which are peculiar to only one of the polarizations is indeed suggestive of orientation effects, but one should not draw conclusions too quickly given the difficulty of estimating the actual influence of the MSSR on the intensities as a function of thickness, and the lack of knowledge about the intrinsic absorption probabilities of the spectroscopic transitions in the crystal. Note for the moment that the peak at $1130 \mathrm{~cm}^{-1}$, not accounted for by the optical indices of Toon et al.,${ }^{15}$ which were derived at $181 \mathrm{~K}$ from transmission experiments sensitive only to the s-polarized component of light, is also very weak in the present s-polarized spectra, as could be expected for vibrations which are almost but not completely oriented in a perpendicular plane to the metal surface. As the crystal thickness grows, the MSSR relaxes, and all transitions have enhanced intensities. In their comparison of transmission spectra of thin $\alpha$-NAT films produced at temperatures between 162 and $180 \mathrm{~K}$, Tisdale et $\mathrm{al}^{23}$ observed that the $1140 \mathrm{~cm}^{-1}$ peak disappeared above $175 \mathrm{~K}$, and indicated that this disappearance was not necessarily due to orientation effects, but could also be explained by changes in the degree of crystallinity of the solid with temperature, that would make the corresponding vibration spectroscopically inactive. To throw some light on this problem, we present in Fig. 3 two spectra recorded in our laboratory. The top frame shows the non-polarized spectrum of the thinnest $(d=370 \mathrm{~nm}) \alpha$-NAT film of Fig. 1 , annealed at $175 \mathrm{~K}$, and the bottom one displays that of a film of approximately the same thickness, but annealed at $180 \mathrm{~K}$. Although 


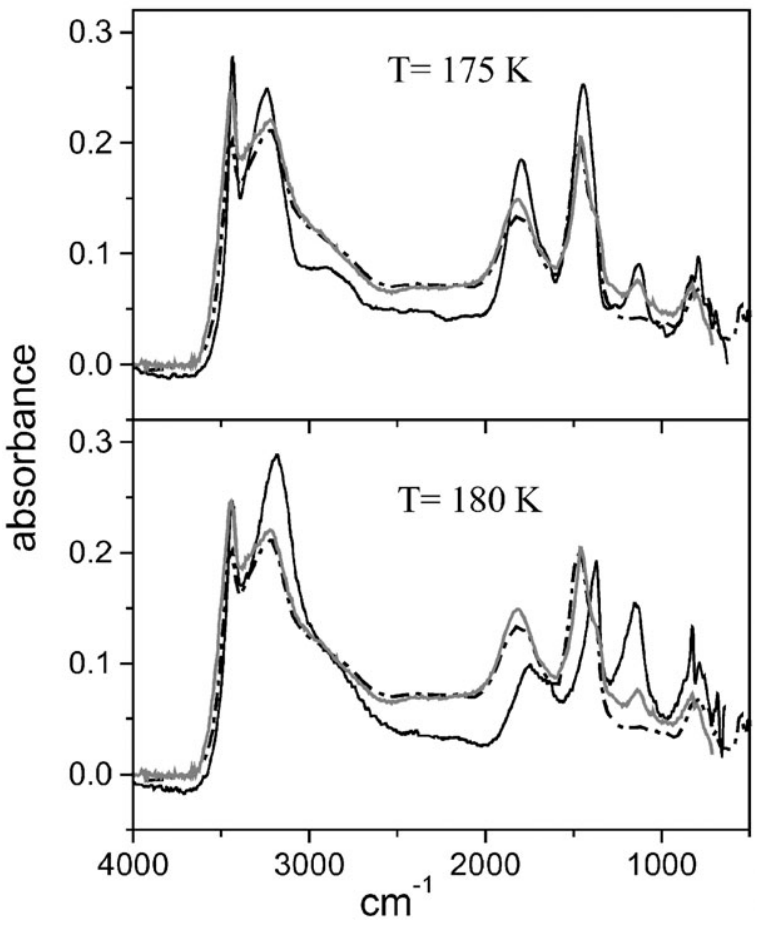

Fig. 3 Solid trace: grazing angle $\left(\theta=75^{\circ}\right)$ non-polarized RAIR spectra of NAT films with a thickness of $370 \mathrm{~nm}$. Upper panel: formed by deposition at $90 \mathrm{~K}$ and annealing at $175 \mathrm{~K}$; lower panel: formed by deposition at $150 \mathrm{~K}$ and annealing at $180 \mathrm{~K}$. Dash-dot trace: simulations of the spectra with a Fresnel model (see text and refs. 27 and 29) and the optical indices of Toon et al. ${ }^{15}$ Gray trace: simulations with the optical indices of Richwine et al. ${ }^{21}$

there are appreciable variations in the relative intensity and shape of some spectral features, which suggest that changes in the sample do indeed take place over this narrow temperature range, the peak at $1140 \mathrm{~cm}^{-1}$ remains and becomes even stronger for the $180 \mathrm{~K}$ sample. Therefore, the hypothesis of Tisdale et al. ${ }^{23}$ does not seem to be confirmed.

It is difficult to derive information about crystal properties from the spectra of the thickest films studied, which are largely distorted by IR interferences. The strong disagreement between measurements and simulations displayed in the two last rows of Fig. 1 may be due to several causes. A failure of the Fresnel model, which implies homogeneous media and flat reflecting surfaces, may be invoked but the same model was successful for the simulation of thicker water-ice films. ${ }^{29}$ An inadequacy of the literature optical indices, as commented on in the previous paragraph, may be another explanation. It is also conceivable that vapor deposition and subsequent annealing might not have led to a homogenous NAT crystalline sample in the case of these thicker layers, in spite of the fact that the RAIR spectra had already stabilized after the $20 \mathrm{~min}$ period of annealing.

RAIR spectra of vapor deposited NAT films, reported by other groups, are shown in Fig. 4, with Fresnel model simulations with the optical indices of Toon et al. ${ }^{15}$ and of Richwine et al. ${ }^{21}$ The simulated spectra have been used as approximate estimates of the thickness where $d$ was not given (see ref. 29). All the films are thin, and the spectra, free from interferences, show the same absorption features, although with different relative intensities. In all cases, the authors assigned the spectra to $\alpha$-NAT in spite of the high temperatures $(>185 \mathrm{~K})$ of some of the samples. The spectrum $4 a$ of Barone et $a l^{32}$ was recorded with p-polarized radiation and with an angle of incidence of $84^{\circ}$ on a very thin film $(10 \mathrm{~nm})$ annealed at $185 \mathrm{~K}$ for a short period of time. The theoretical simulations for $d=10 \mathrm{~nm}$, which is the thickness reported by the authors, are in reasonable agreement with the absorbance measured

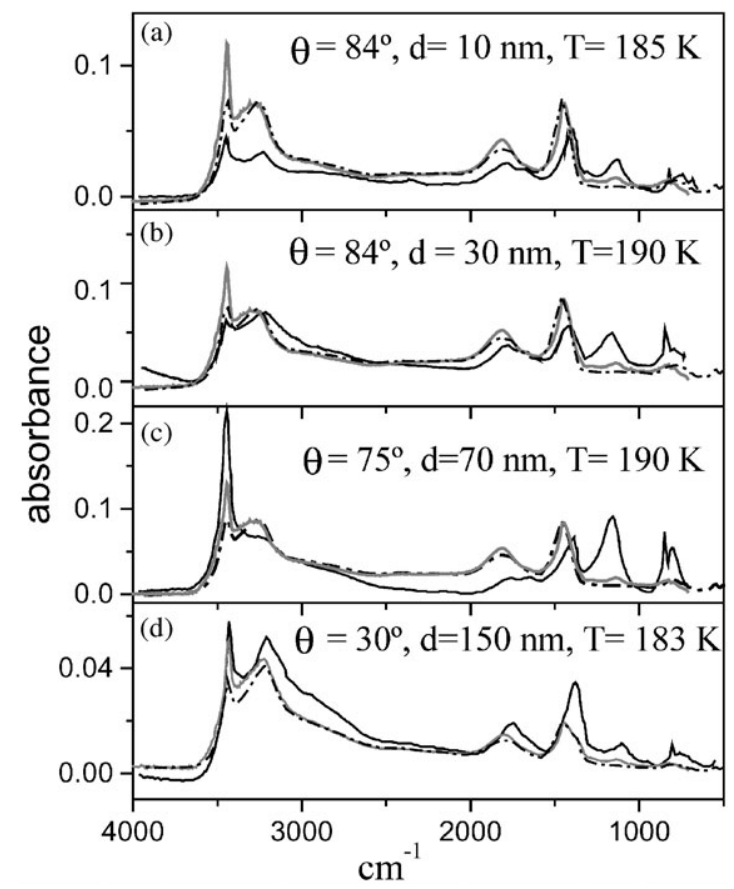

Fig. 4 RAIR spectra (solid lines) of thin NAT films from the literature and Fresnel simulations with the optical indices of Toon et $a .^{15}$ (dash-dot trace) and of Richwine et $a l^{21}$ (gray trace). Panel $a$ : experimental data from Barone et al. ${ }^{32}$ Angle of incidence $84^{\circ}$, experimental thickness $10 \mathrm{~nm}$. Panel $b$ : experimental data from Hudson et al. ${ }^{33}$ Angle of incidence $84^{\circ}$, simulated thickness $30 \mathrm{~nm}$. Panel $c$ : experimental data from Koch et al. ${ }^{14}$ Angle of incidence $75^{\circ}$, simulated thickness $70 \mathrm{~nm}$. Panel $d$ : experimental data from Tso and Leu. ${ }^{13}$ Angle of incidence $30^{\circ}$, simulated thickness $150 \mathrm{~nm}$.

below $3000 \mathrm{~cm}^{-1}$, but overestimate the signal, especially in the region of the $\mathrm{OH}$ stretching around $3000-3500 \mathrm{~cm}^{-1}$. The spectrum $4 b$ of Hudson et al. ${ }^{33}$ corresponds to a thin layer of NAT generated at $190 \mathrm{~K}$ and recorded with non-polarized radiation at the same angle of incidence; no estimate of the thickness was provided in this work. The model gives a reasonably good simulation of the data with $d=30 \mathrm{~nm}$. The spectrum $4 c$ was measured by Koch et al. ${ }^{14}$ on a thin NAT film annealed at $190 \mathrm{~K}$ using non-polarized IR radiation at an angle of incidence of $75^{\circ}$. Simulations with $d=70 \mathrm{~nm}$, not too far from the $100 \mathrm{~nm}$ value estimated by the authors, led to a reasonable agreement with the measured spectral shape (in this case the authors did not provide absolute absorbance values) for wavenumbers lower than $3500 \mathrm{~cm}^{-1}$, but the model cannot account for the sharp peak at $3500 \mathrm{~cm}^{-1}$. The spectrum $4 d$ has been taken from Fig. 4 of Tso and Leu. ${ }^{13}$ For homogeneity the original base e absorbance units have been transformed to base 10 . In this study, the incidence angle of the IR radiation was $30^{\circ}$, rather different from the usual near grazing incidence used in most RAIR experiments. For a given NAT film thickness, this geometry, which is closer to normal incidence, leads to smaller signals at lower wavenumbers, as compared with a grazing angle arrangement. This effect is accounted for by the Fresnel model that reproduces approximately the measured spectrum with an assumed thickness of $150 \mathrm{~nm}$. In their article Tso and Leu reported absortivity values that would lead to a thickness of $\approx 74 \mathrm{~nm}$. Both sets of optical indices give very similar simulations and consequently a similar level of discrepancy with the measurements. For the thin films displayed in Fig. 4, the simulations with the indices of Richwine et al. ${ }^{21}$ provide only a hint of the signal at 1140 $\mathrm{cm}^{-1}$ and are much closer to the absorbance values predicted with the indices of Toon et al. ${ }^{15}$ than to the actual experimental peaks.

Although the spectra of Fig. 4 cannot be strictly compared to those of the present work, since they correspond to thinner 
films and only one of them was recorded with an angle of incidence of $75^{\circ}$, the same absorption features are easily identifiable. In the grazing angle spectra of Fig. 4, the pattern of the three bands between 2000 and $1000 \mathrm{~cm}^{-1}$ resembles more our spectrum at $180 \mathrm{~K}$ than the one at $175 \mathrm{~K}$ (see Fig. 3). Note that in all the RAIR spectra for $T \geq 180 \mathrm{~K}$ (Fig. 3, bottom, and Fig. 4) measured with either p-polarized or nonpolarized radiation, the peak at $\approx 1130 \mathrm{~cm}^{-1}$ is present, in contrast with the behavior observed by Tisdale et $a l^{23}$ in transmission spectra of NAT films at $180 \mathrm{~K}$. These results suggest strongly that orientation effects, and not only structural changes induced by the temperature rise, are responsible for the observations. We are only aware of a published RAIR spectrum, corresponding to an $\alpha$-NAT film produced at $175 \mathrm{~K}^{31}$ which does not show the peak at $\approx 1140 \mathrm{~cm}^{-1}$. However an assessment of this result is very difficult since in that work neither the angle of incidence, nor the polarization state of the light, or an estimate of the film thickness, were provided. Inspection of the spectra presented in Fig. 4 reveals that the peak under discussion is weakest in the spectrum at closest to normal incidence illumination, which is consistent with the interpretation proposed in this work.

Orientation effects on very thin films of NAT were addressed by Sodeau and co-workers. ${ }^{14}$ To this aim they used the grazing angle RAIR spectrum of Fig. 4c, and a normal incidence transmission spectrum of a similar NAT film. In the RAIR spectrum of this very thin film $(d<100 \mathrm{~nm})$ the MSSR is expected to suppress largely the intensity of transitions caused by the s- component of the radiation, i.e. those corresponding to molecular vibrations inducing a dipole moment preferentially aligned parallel to the substrate plane. In contrast, the intensity of these transitions will be favored in the transmission spectrum (a graphic representation of the vibrational modes of the nitrate and hydronium ions can be found in Fig. 4 of ref. 14). From a comparison of the two spectra the authors concluded that the nitrate and oxonium ions in the NAT film align preferentially with their $C_{3}$ axes parallel to each other and perpendicular to the underlying substrate, and pointed out that this arrangement was apparently consistent with the crystal structure of NAT. Below $2000 \mathrm{~cm}^{-1}$, Koch et al. ${ }^{14}$ noted that for this orientation, spectra corresponding to s-polarized radiation should be dominated by peaks at about 1765 and $1390 \mathrm{~cm}^{-1}$, assigned to $v_{4}\left(\mathrm{H}_{3} \mathrm{O}^{+}\right)$and $v_{3}\left(\mathrm{NO}_{3}{ }^{-}\right)$respectively, whereas in spectra corresponding to p-polarized light, the absorptions at $\approx 1136 \mathrm{~cm}^{-1}$ and $821 \mathrm{~cm}^{-1}$, assigned to $v_{2}\left(\mathrm{H}_{3} \mathrm{O}^{+}\right)$and $v_{2}\left(\mathrm{NO}_{3}{ }^{-}\right)$respectively, should prevail. Below $2000 \mathrm{~cm}^{-1}$, all the s-polarized spectra of the present work as well as the literature transmission spectra of NAT films $\mathrm{s}^{6,7,9,10,23,40}$ and aerosols ${ }^{19-22}$ are indeed dominated by two peaks at about the mentioned frequencies. However, for the p-polarization, the absorptions at the two lower frequencies are not the strongest in general even for very thin films ${ }^{13,14,32,33}$ (see Figs. 1, 4 and 5). In most cases a peak at about $1400 \mathrm{~cm}^{-1}$ is the most intense one for the two types of polarization.

A recent publication ${ }^{37}$ reports a calculation of the refined structure and vibrational spectrum of a NAT crystal by density functional theory (DFT) methods. Two views of the calculated structure (from the $a b$ and $b c$ unit-cell planes), which is in very good agreement with the X-ray data ${ }^{11,12}$, are represented in Fig. 5. This figure shows that the symmetry axes of the four hydronium ions in the unit cell are nearly parallel and aligned almost perpendicularly to the $a b$ plane. In contrast, the four $\mathrm{NO}_{3}{ }^{-}$ions lie in slightly different planes and their symmetry axes are not parallel to each other nor to those of the $\mathrm{H}_{3} \mathrm{O}^{+}$ ions, but they all form a fairly large angle with the $a b$ plane. An orientation of the NAT polycrystals with their $a b$ plane approximately parallel to the substrate would thus be relatively close to the situation described by Sodeau and co-workers ${ }^{14}$ for their thin films, and would be coincident with the orientation suggested by the comparison of the $632 \mathrm{~nm}$ refractive indices

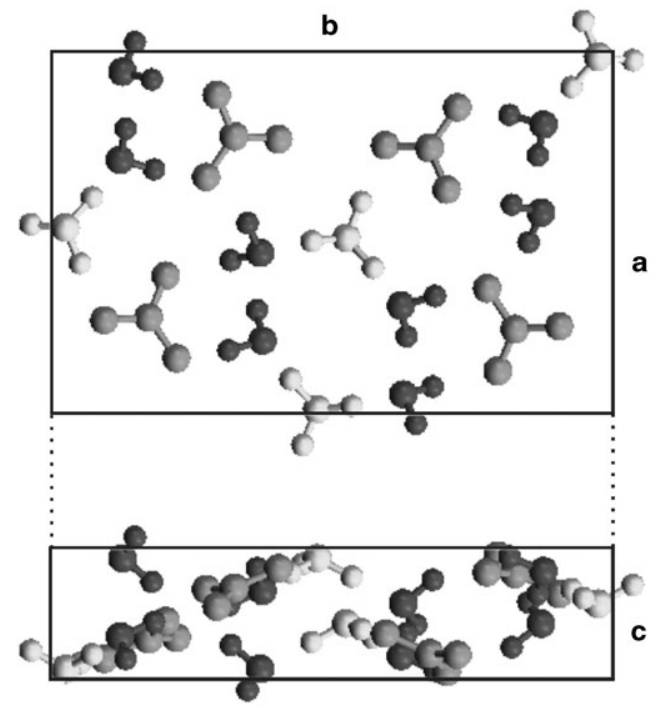

Fig. 5 Atomic arrangement within the $\beta$-NAT unit cell $(a=9.48 \AA$, $b=14.68 \AA$ and $c=3.43 \AA$ ). For a better appreciation the various molecular units are represented with different shades. White: hydronium ions; gray: nitrate ions; dark gray: water molecules.

measured in the experiments of Middlebrook et al. ${ }^{40}$ and of Koosmanian et $a .^{24}$ (see above). A division of the vibrational modes of the ions into "s-active" and "p-active" might still be useful, although the separation would not be entirely clear-cut.

\section{Assignments}

In ref. 37 an assignment of the main molecular vibrations corresponding to the crystal normal modes between 3500 and $1000 \mathrm{~cm}^{-1}$ was presented. Previous empirical assignments ${ }^{7,8,13}$ were in general corroborated, but a significant mixing of molecular vibrations, sometimes from non-equivalent molecules within the unit cell, was found in many of the calculated normal modes. In the present work the theoretical assignment is extended down to $650 \mathrm{~cm}^{-1}$. The predominant molecular vibrations corresponding to the crystal vibrational frequencies between 2000 and $650 \mathrm{~cm}^{-1}$ are listed in Table 2 . Below $\approx$ $1000 \mathrm{~cm}^{-1}$ lattice vibrations, usually hindered rotations of $\mathrm{H}_{2} \mathrm{O}$ and $\mathrm{H}_{3} \mathrm{O}^{+}$units, contribute appreciably to the crystal normal modes; these vibrations are globally labeled as $v_{\mathrm{L}}$ in the table.

A rigorous comparison between experiment and theory would require a calculation not only of the frequencies, but also of the intensities of the crystal normal modes, and a proper

Table 2 Assignment of the $\alpha$-NAT spectrum ${ }^{a}$ in the $2000-650 \mathrm{~cm}^{-1}$ wavenumber range

\begin{tabular}{ll}
\hline Predominant vibrations & Wavenumber range $/ \mathrm{cm}^{-1}$ \\
\hline$v\left(\mathrm{H}_{5} \mathrm{O}_{2}^{+}\right)$ & $1897-1800(4)$ \\
$v_{4}\left(\mathrm{H}_{3} \mathrm{O}^{+}\right)+v_{2}\left(\mathrm{H}_{2} \mathrm{O}\right)$ & $1707-1651(8)$ \\
$v_{2}\left(\mathrm{H}_{2} \mathrm{O}\right)$ & $1610-1593(4)$ \\
$v_{2}\left(\mathrm{H}_{2} \mathrm{O}\right)+v_{3}\left(\mathrm{H}_{3} \mathrm{O}^{+}\right)$ & $1576-1560(4)$ \\
$v_{3}\left(\mathrm{NO}_{3}^{-}\right)$ & $1476-1369(8)$ \\
$v_{2}\left(\mathrm{H}_{3} \mathrm{O}^{+}\right)+v_{2}\left(\mathrm{H}_{2} \mathrm{O}\right)$ & $1319-1306(4)$ \\
$v_{1}\left(\mathrm{NO}_{3}^{-}\right)$ & $1081-1071(4)$ \\
$v_{\mathrm{L}}+v_{1}\left(\mathrm{NO}_{3}^{-}\right)$ & $1057-1017(4)$ \\
$v_{\mathrm{L}}$ & $924-835(16)$ \\
$v_{2}\left(\mathrm{NO}_{3}^{-}\right)$ & $766-764(4)$ \\
$v_{\mathrm{L}}+v_{4}\left(\mathrm{NO}_{3}^{-}\right)+v_{2}\left(\mathrm{NO}_{3}^{-}\right)$ & $747-714(6)$ \\
$v_{4}\left(\mathrm{NO}_{3}^{-}\right)+v_{\mathrm{L}}$ & $712-647(14)$
\end{tabular}

${ }^{a}$ The number of fundamental normal modes contributing to each vibration and wavenumber range is indicated in parentheses. 


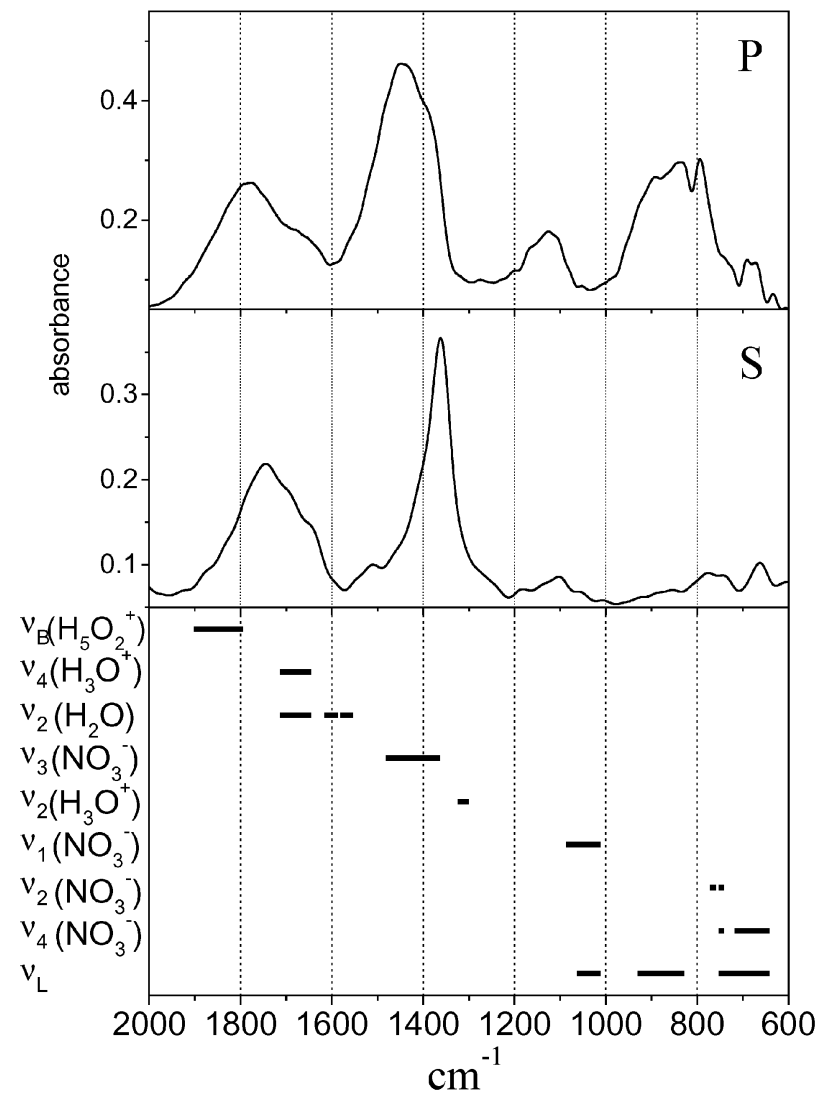

Fig. 6 Upper part: Enlargement of the p- and s-polarized spectra of the bottom panel of Fig. 2 (corresponding to an $800 \mathrm{~nm}$ film deposited at $90 \mathrm{~K}$ and annealed at $175 \mathrm{~K}$ ) in the $2000-650 \mathrm{~cm}^{-1}$ wavenumber range. Lower part: theoretical assignment indicating wavenumber range of each vibrational mode (see also Table 2).

simulation of the RAIR spectra for different orientations. As far as we know this information is not yet available, but still some insight can be gained from the consideration of the spectral assignment (Table 2) in relation to a likely orientation. In the following, it will be assumed that the NAT polycrystals are oriented with their $a b$ faces parallel to the substrate, which is the closest approach to the orientation suggested in the work of Koch et al. ${ }^{14}$ Fig. 6 shows an enlargement of the polarized spectra of the NAT film with $d=800 \mathrm{~nm}$ (see Fig. 2) in the 2000 to $650 \mathrm{~cm}^{-1}$ wavenumber range. These spectra are free from interference perturbations and correspond to a thickness for which the MSSR should be at least partially relaxed. The spectra consist of four bands, which exhibit different polarization behavior. In order to facilitate this discussion the calculated spectral ranges corresponding to the different molecular vibrations within the crystal are indicated in the lower part of the Figure. According to this calculation, the higher frequency band $\left(\approx 1900-1600 \mathrm{~cm}^{-1}\right)$ is a composition of the asymmetric bending of the oxonium ion, $v_{4}\left(\mathrm{H}_{3} \mathrm{O}^{+}\right)$, as assigned in refs. 7,8,14 and 23 , the bending mode of water $v_{2}\left(\mathrm{H}_{2} \mathrm{O}\right)$ as indicated in ref. 13, and also the "H-bridging" mode ${ }^{37}$ $v_{\mathrm{B}}\left(\mathrm{H}_{5} \mathrm{O}_{2}^{+}\right)$. The first of these vibrations induces a change in the dipole moment along the $a b$ plane, and should only be active for the s-polarization, but the other two vibrations would also lead to absorptions with p-polarized light. This would explain the observation of this peak in both kinds of spectra.

The most intense experimental band in this region, with a maximum at about $1400 \mathrm{~cm}^{-1}$ is usually attributed in the literature to the asymmetric stretch of the nitrate ion, ${ }^{7,8,13,14,23}$ which should be mainly, but not exclusively, s-active for the orientation assumed. The calculations predict this mode between 1369 and $1476 \mathrm{~cm}^{-1}$ in good agreement with the range of the intense absorption recorded in the s-polarized spectrum. In addition, the predictions extend the above mentioned $v_{2}\left(\mathrm{H}_{2} \mathrm{O}\right)$ mode down to $1560 \mathrm{~cm}^{-1}$ and locate $v_{2}\left(\mathrm{H}_{3} \mathrm{O}^{+}\right)$ at the lower band edge of this region. The latter mode should contribute to the p-polarized spectra and the former to both polarizations. The larger width of the p-polarized band could therefore be explained by contributions from all three modes.

The controversial band between 1200 and $1080 \mathrm{~cm}^{-1}$, with a maximum at about $1130 \mathrm{~cm}^{-1}$ is predominantly p-active. This feature is usually assigned to $v_{2}\left(\mathrm{H}_{3} \mathrm{O}^{+}\right){ }^{7,14,23}$ but Tso and $\mathrm{Leu}^{13}$ attribute it to $v_{1}\left(\mathrm{NO}_{3}{ }^{-}\right)$. The theoretical calculations ${ }^{37}$ would favor this latter assignment predicted between 1071 and $1081 \mathrm{~cm}^{-1}$, whereas $v_{2}\left(\mathrm{H}_{3} \mathrm{O}^{+}\right)$is calculated at higher frequencies, but given the quasi ternary internal symmetry of $\mathrm{NO}_{3}{ }^{-}$ within the crystal, ${ }^{11,12,37}$ this mode should be almost inactive. The discrepancy could be explained on two different bases. First, due to an inaccuracy of the theoretical results yielding too high frequencies for $v_{2}\left(\mathrm{H}_{3} \mathrm{O}^{+}\right)$, and second in terms of structural considerations. The calculations are based on the only NAT structure available thus far, ${ }^{11,12}$ derived from measurements carried out on NAT samples crystallized at high temperature $(>200 \mathrm{~K})$. Recent evidence ${ }^{46}$ indicates that this $\mathrm{X}$-ray structure corresponds to the more stable, higher temperature $\beta$-NAT phase, rather than to the $\alpha$-NAT phase formed in this study. Whereas the frequency ranges associated with the various molecular absorptions should not be very different in the two phases, ${ }^{10,13}$ some changes in the crystal structure could induce a slight distortion of the symmetry of the $\mathrm{NO}_{3}{ }^{-}$ions in the lattice that would render the $v_{1}$ mode p-active. In the absence of more data one can only speculate on this point, but the orientation suggested in this discussion would be consistent with either of the two possible assignments. In this respect, the recent experiments by the group of Grothe and co-workers, ${ }^{46-48}$ which combine X-ray and IR spectroscopy for the study of comparable samples could be very clarifying.

The band between 980 and $700 \mathrm{~cm}^{-1}$, which is also predominantly p-active, contains a large number of modes corresponding to lattice vibrations. At about $765 \mathrm{~cm}^{-1}$, in coincidence with a narrow peak on top of the band, the calculations predict the p-active symmetric bending of the nitrate ion, $v_{2}\left(\mathrm{NO}_{3}{ }^{-}\right)$. Finally, the asymmetric bending $v_{4}\left(\mathrm{NO}_{3}{ }^{-}\right)$, with a marked s-character, is predicted in the region of the small band observed between $\approx 650$ and $700 \mathrm{~cm}^{-1}$. The relative intensity of this absorption feature compared with the adjoining band is clearly higher in the s-polarized spectrum than in the p-polarized one.

The above assignment considerations are only of a qualitative nature. In particular, it is very difficult to estimate the polarization activity of the modes implying bending vibrations of water or lattice vibrations, based on the inspection of the atomic displacements per normal mode used in our works. ${ }^{37}$ In general the observations of the previous paragraph are consistent with the preferential orientation of the $a b$ crystallographic plane parallel to the deposition surface even for values of the film thickness of $800 \mathrm{~nm}$, but little more can be said before proper dipole moment intensity calculations can be performed. The moderate increase in temperature from 175 to $180 \mathrm{~K}$ seems to have a pronounced influence on the alignment of the crystals with respect to the substrate, reflected by an enhancement of the intensity differences between $\mathrm{p}$ - and s-active absorption features. In the grazing-angle RAIR spectra of comparatively thin films, this leads to a decrease of the bands with s-active vibrations (due to the MSSR) and to a concomitant growth in the bands with preponderant $\mathrm{p}$-active vibrations (see Fig. 3). In transmission spectra recorded with normal incidence, the bands dominated by p-active vibrations would experience a marked decline in intensity as observed in ref. 23. 


\section{Summary and conclusions}

Grazing angle RAIR spectra of $\alpha$-NAT films with thickness between $370 \mathrm{~nm}$ and $2 \mu \mathrm{m}$ formed by vapor deposition and subsequent annealing on an Al substrate, have been recorded using s-polarized, p-polarized and non-polarized IR radiation. In all cases distinct spectra were obtained for the two polarizations employed. A simulation of the spectra with thickness lower than $1 \mu \mathrm{m}$ using a classical Fresnel model ${ }^{27,29}$ and empirical optical indices from the literature ${ }^{15,21}$ could account for the location of the main spectral features but failed often to reproduce the measured absorbance, especially in the p-polarized and non-polarized spectra. For films thicker than $1 \mu \mathrm{m}$, where IR interferences are relevant, noteworthy disagreements, not only in the intensity, but also in the location of the peaks were obtained between measurements and simulations. In a previous work, the same model was applied to films of water ice with thickness values ranging from $100 \mathrm{~nm}$ to more than $5 \mu \mathrm{m}$, and a satisfactory simulation, including the interference pattern, was obtained. This contrasting behavior may be attributed to the different crystal structures of NAT and water. The orthorhombic lattice of NAT, with appreciably different values for the three unit-cell dimensions, will likely exhibit much more anisotropic properties than the disordered cubic or hexagonal lattice of ice, like optical birefringence ${ }^{24}$ or a tendency to a preferential alignment when formed on a surface. ${ }^{14}$ It is thus possible that the procedures used for the derivation of the empirical optical indices, which use models that assume isotropic media, are adequate for water ice, ${ }^{15,49}$ but yield inaccurate results for NAT films. ${ }^{15,21}$ Moreover it is possible that each sample be characterized by a somewhat different set of optical indices depending on the degree of crystallinity and on the degree of orientation.

A comparison of the spectra under conditions free from IR interferences (i.e. for wavenumbers between 2000 and $650 \mathrm{~cm}^{-1}$ and for thickness values up to $800 \mathrm{~nm}$ ) with the theoretical assignment of vibrational modes of the NAT crystal, ${ }^{37}$ is consistent with a preferential alignment of the unit-cell $a b$ face parallel to the deposition surface, but a firm conclusion cannot be reached before reliable calculations of the intensity of the vibrational modes of the crystal become available. This proposed geometry implies that the $C_{3}$ symmetry axis of the nitrate and hydronium ions form large angles with respect to the substrate, in accordance with the orientation suggested by Sodeau and co-workers ${ }^{14}$ for very thin $(\approx 100 \mathrm{~nm})$ films of NAT. For this orientation the vibrational normal modes of the ions should exhibit a marked selectivity for the interaction with either $\mathrm{p}$ - or s-polarized light, reflected in large differences in shape and intensity of the corresponding bands. In the present experiments none of the bands disappears completely with a change in the polarization of the light, due probably to an incomplete orientation of the crystals, and to the mixing of various molecular modes in the observed absorption features.

In their study, based on normal incidence transmission spectra, Tisdale et al. ${ }^{23}$ observed the gradual disappearance of the peak at $\approx 1140 \mathrm{~cm}^{-1}$ when the temperature was increased from 175 to $180 \mathrm{~K}$ and suggested that it could be due to changes in the crystal structure. Significant variations take place also in the RAIR spectra for $T \geq 180 \mathrm{~K}$ which are indicative of appreciable changes in the sample. However, the results point rather to a rearrangement of the polycrystals than to a change in the microscopic structure. In fact, the peak at $1140 \mathrm{~cm}^{-1}$ is present in all the p-polarized or non-polarized RAIR spectra irrespective of the temperature of the sample, and is even enhanced in the higher temperature spectra, which suggests that a macroscopic reorientation has taken place favoring p-polarized vibrations. Although the usual empirical assignments attribute this peak to the $v_{2}\left(\mathrm{H}_{3} \mathrm{O}^{+}\right)$mode, the theoretical results are not clear on this point. Further experimental and theoretical work is needed in order to improve the understanding of the relation between structure and spectra of NAT crystals.

\section{Acknowledgements}

This investigation has been funded by the Spanish Ministry of Science and Technology, Project REN2000-1557. The work has been carried out within the framework of the Unidad Asociada CSIC-Universidad de Jaén. We are grateful to D. Fernández for her help in the theoretical assignments, and to J.M. Castillo and J. Rodríguez for technical help.

\section{References}

1 S. Solomon, Rev. Geophys., 1999, 106, 275.

2 C. Voigt, J. Schreiner, A. Kohlmann, P. Zink, K. Mauersberger, N. Larsen, T. Deshler, C. Kröger, J. Rosen, A. Adriani, F. Cairo, G. Di Donfrancesco, M. Viterbini, J. Ovarlez, H. Ovarlez, C. David and A. Dörnbrack, Science, 2000, 290, 1756.

3 M. A. Zondlo, P. K. Hudson, A. J. Prenni and M. A. Tolbert, Annu. Rev. Phys. Chem., 2000, 51, 473.

4 D. W. Fahey, R. S. Gao, K. S. Carslaw, J. Kettleborough, P. J. Popp, M. J. Northway, J. C. Holecek, S. C. Ciciora, R. J. McLaughlin, T. L. Thompson, R. H. Winkler, D. G. Baumgardner, B. Gandrud, P. O. Wennberg, S. Dhaniyala, K. McKinney, Th. Peter, R. J. Salawitch, T. P. Bui, J. W. Elkins, C. R. Webster, E. L. Atlas, H. Jost, J. C. Wilson, R. L. Herman, A. Kleinböhl and M. von König, Science, 2001, 291, 1026.

5 M. A. Tolbert and O. B. Toon, Science, 2001, 292, 61.

6 M. A. Tolbert and A. M. Middlebrook, J. Geophys. Res., 1990, 95, 22423.

7 G. Ritzhaupt and J. P. Devlin, J. Phys. Chem., 1991, 95, 90.

8 R. H. Smith, M.-T. Leu and L. F. Keyser, J. Chem. Phys., 1991, 95, 5924

9 M. A. Tolbert, B. G. Koehler and A. M. Middlebrook, Spectrochim. Acta, 1992, 48A, 1303.

10 B. G. Koehler, A. M. Middlebrook and M. A. Tolbert, J. Geophys. Res., 1992, 97, 8065.

11 I. Taesler, R. G. Delaplane and I. Olovsson, Acta Crystallogr., 1975, B31, 1489.

12 N. Lebrun, F. Mahe, J. Lamiot, M. Foulon, J. C. Petit and D. Prevost, Acta Cryst. B, 2001, 57, 27.

13 T.-L. Tso and M.-T. Leu, Anal. Sci., 1996, 12, 615.

14 T. G. Koch, N. S. Holmes, T. B. Roddis and J. R. Sodeau, J. Chem. Soc., Faraday Trans., 1996, 92, 4787.

15 O. B. Toon, M. A. Tolbert, B. G. Koehler, A. M. Middlebrook and J. Jordan, J. Geophys. Res., 1994, 99(D12), 25631.

16 S. Kinne, O. B. Toon, G. C. Toon, C. B. Farmer, E. V. Browell and M. P. McCormick, J. Geophys. Res., 1989, 94, 16481.

17 G. C. Toon, C. B. Farmer, L. L. Lowess, P. W. Schaper, J. F. Blavier and R. H. Norton, J. Geophys. Res., 1989, 94, 16571.

18 O. B. Toon and M. A. Tolbert, Nature, 1995, 375, 218.

19 N. Barton, B. Rowland and J. P. Devlin, J. Phys. Chem., 1993, 97, 5848 .

20 R. Callagan, I. J. Lim, D. E. Murdock and J. J. Sloan, Geophys. Res. Lett., 1994, 22, 373.

21 J. L. Richwine, M. L. Clapp, R. E. Miller and D. R. Worsnop, Geophys. Res. Lett., 1995, 22, 2625.

22 A. K. Bertram and J. J. Sloan, J. Geophys. Res. D, 1998, 103, 13261.

23 R. T. Tisdale, A. J. Prenni, L. T. Iraci, M. A. Tolbert and O. B. Toon, Geophys. Res. Lett., 1999, 26, 707.

24 D. Kooshmanian, F. Domine and P. Baldeck, in Polar Stratospheric Ozone: preceedings of the third European workshop, eds. J. A. Pyle, N. R. P. Harris, G. T. Amnatidis, Office for Official Publications of the European Communities, Luxembourg, 1996, p. 762.

25 R. Wagner, A. Mangold, O. Möhler, H. Saathoff and U. Schurath, Atmos. Chem. Phys., 2003, 3, 1147.

26 R. G. Greenler, J. Chem. Phys., 1966, 44, 610.

27 J. D. E. McIntyre and D. E. Aspens, Surf. Sci., 1970, 24, 417.

28 W. Suëtaka, Surface Infrared and Raman Spectroscopy: Methods and Applications, Plenum, New York and London, 1995.

29 B. Maté, A. Medialdea, M. A. Moreno, R. Escribano and V. J. Herrero, J. Chem. Phys. B, 2003, 107, 11098.

30 S. Mitlin and K. T. Leung, J. Phys. Chem. B, 2002, 106, 6234

31 S. Peil, S. Seisel and O. Schrems, J. Mol. Struct., 1995, 348, 449

32 S. B. Barone, M. A. Zondlo and M. A. Tolbert, J. Phys. Chem. A, 1997, 101, 8643 
33 P. K. Hudson, M. A. Zondlo and M. A. Tolbert, J. Phys. Chem. A, 2002, 106, 2882.

34 M. Sato, O. Setokuchi, K. M. T. Yamada and T. Ibusuki, Vib. Spectros., 2003, 37, 167.

35 H. Reinhardt, M. Fida and R. Zellner, J. Mol. Struct., 2003, 567, 661-662.

36 R. Escribano, M. Couceiro, P. C. Gómez, E. Carrasco, M. A. Moreno and V. J. Herrero, J. Phys. Chem. A, 2003, 107, 651.

37 D. Fernández, V. Botella, V. J. Herrero and R. Escribano, J. Phys. Chem. B, 2003, 107, 10608.

38 E. Carrasco, J. M. Castillo, R. Escribano, V. J. Herrero, M. A. Moreno and J. Rodríguez, Rev. Sci. Instrum., 2002, 73, 3469.

39 I. K. Ortega, R. Escribano, D. Fernández, V. J. Herrero, B. Maté, A. Medialdea and M. A. Moreno, Chem. Phys. Lett., 2003 378, 218.

40 A. M. Middlebrook, B. S. Berland, S. M. George and M. A. Tolbert, J. Geophys. Res., 1994, 99, 25665.
41 D. E. Brown, S. M. George, C. Huang, E. K. L. Wong, K. B. Rider, R. S. Smith and B. D. Kay, J. Phys. Chem., 1996, 100, 5460.

42 S. A. Stern and J. T. Mullhaupt, Chem. Rev., 1960, 60, 185.

43 Handbook of the Optical Constants of Solids, ed. E. D. Palik, Academic, Toronto, 1985.

44 M. S. Robinson, G. Mallick, J. L. Spillman, P. A. Carreon and S. Shalloo, Appl. Opt., 1999, 38(1), 91

45 R. F. Niedziela, R. E. Miller and D. R. Worsnop, J. Phys. Chem. $A, 1998,102,6477$.

46 H. Tizek, E. Knözinger and H. Grothe, Phys. Chem. Chem. Phys., 2004, 6, 972.

47 H. Tizek, E. Knözinger and H. Grothe, Phys. Chem. Chem. Phys., 2002, 4, 5128.

48 H. Grothe, C. E. L. Myhre and H. Tizek, Vib. Spectrosc., 2004, 34(1), 55.

49 M. L. Clapp, R. E. Miller and D. R. Worsnop, J. Phys. Chem., 1995, 99, 6317. 